\title{
RESEARCH
}

Open Access

\section{Sex-specific associations with cerebrospinal fluid biomarkers in dementia with Lewy bodies}

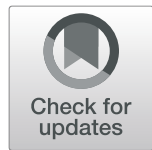

M. van de Beek ${ }^{1 *+}$, R. Babapour Mofrad ${ }^{1,2+}$, I. van Steenoven ${ }^{1,2}$, H. Vanderstichele ${ }^{3}$, P. Scheltens ${ }^{1}$, C. E. Teunissen ${ }^{2}$, A. W. Lemstra ${ }^{1}$ and W. M. van der Flier ${ }^{1,4}$

\begin{abstract}
Background: Dementia with Lewy bodies (DLB) is more prevalent in men than in women. In addition, postmortem studies found sex differences in underlying pathology. It remains unclear whether these differences are also present antemortem in in vivo biomarkers, and whether sex differences translate to variability in clinical manifestation. The objective of this study was to evaluate sex differences in cerebrospinal fluid (CSF) biomarker concentrations (i.e., alpha-synuclein ( $\alpha$-syn), amyloid $\beta 1-42$ (Aß42), total tau (Tau), phosphorylated tau at threonine 181 (pTau)) and clinical characteristics in DLB.
\end{abstract}

Methods: We included 223 DLB patients from the Amsterdam Dementia Cohort, of which 39 were women (17\%, age $70 \pm 6$, MMSE $21 \pm 6$ ) and 184 men (83\%, age $68 \pm 7$, MMSE $23 \pm 4$ ). Sex differences in CSF biomarker concentrations (i.e., $\mathrm{a}-\mathrm{syn}, \mathrm{A} 42 \mathrm{2}$, Tau, and pTau) were evaluated using age-corrected general linear models (GLM). In addition, we analyzed sex differences in core clinical features (i.e., visual hallucinations, parkinsonism, cognitive fluctuations, and REM sleep behavior disorder (RBD) and cognitive test scores using age- and education-adjusted GLM.

Results: Women had lower CSF a-syn levels (F $1429 \pm 164$ vs M $1831 \pm 60, p=0.02$ ) and CSF Aß42 levels (F $712 \pm 39$ vs M $821 \pm 18, p=0.01)$ compared to men. There were no sex differences for ( $p)$ Tau concentrations $(p>0.05)$. Clinically, women were older, had a shorter duration of complaints ( $F 2 \pm 1$ vs M $4 \pm 3, p<0.001$ ), more frequent hallucinations ( $58 \%$ vs $38 \%, p=0.02$ ), and scored lower on MMSE and a fluency task (MMSE, $p=0.02$; animal fluency, $p=0.006$ ). Men and women did not differ on fluctuations, RBD, parkinsonism, or other cognitive tests.

\footnotetext{
* Correspondence: m.vandebeek2@amsterdamumc.nl

${ }^{\dagger} \mathrm{M}$. van de Beek and R. Babapour Mofrad contributed equally to this work. ${ }^{1}$ Alzheimer Center Amsterdam \& Department of Neurology, Neuroscience Campus Amsterdam Neuroscience, Vrije Universiteit Amsterdam, Amsterdam UMC, VU University Medical Center, Amsterdam, The Netherlands Full list of author information is available at the end of the article
}

(c) The Author(s). 2020 Open Access This article is licensed under a Creative Commons Attribution 4.0 International License, which permits use, sharing, adaptation, distribution and reproduction in any medium or format, as long as you give appropriate credit to the original author(s) and the source, provide a link to the Creative Commons licence, and indicate if changes were made. The images or other third party material in this article are included in the article's Creative Commons licence, unless indicated otherwise in a credit line to the material. If material is not included in the article's Creative Commons licence and your intended use is not permitted by statutory regulation or exceeds the permitted use, you will need to obtain permission directly from the copyright holder. To view a copy of this licence, visit http://creativecommons.org/licenses/by/4.0/ The Creative Commons Public Domain Dedication waiver (http://creativecommons.org/publicdomain/zero/1.0/) applies to the data made available in this article, unless otherwise stated in a credit line to the data. 


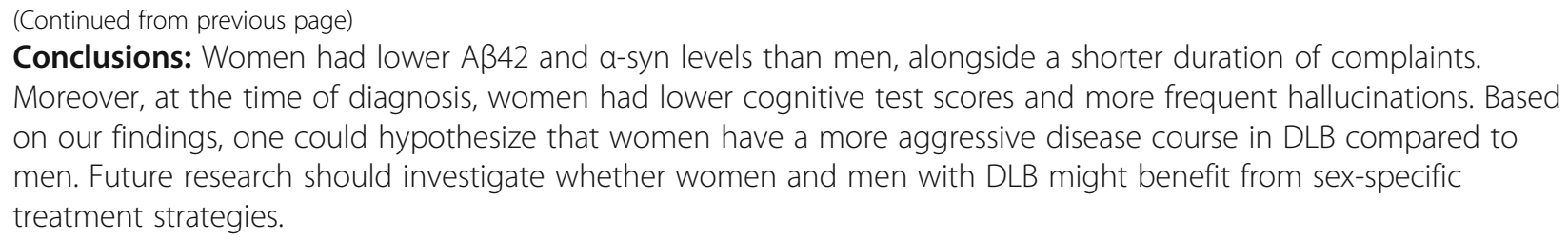

Keywords: Dementia with Lewy bodies, Sex differences, Cerebrospinal fluid biomarkers

\section{Background}

Dementia with Lewy bodies (DLB) is clinically characterized by cognitive decline, visual hallucinations, parkinsonism, cognitive fluctuations, and rapid eye movement (REM) sleep behavior disorder (RBD) [1]. Pathologically, DLB is characterized by the presence of cortical Lewy bodies, i.e., neuronal inclusions of alpha-synuclein proteins [2], frequently combined with Alzheimer's disease (AD) pathology, i.e., amyloid plaques and neurofibrillary tangles (NFT) [3]. Most studies report a higher prevalence of DLB in men than in women [4-7], although a few report the opposite $[8,9]$. In addition to the skewed distribution, there is evidence of differences in the underlying pathology between women and men with DLB. Post-mortem examinations showed that men were more likely to die from "pure" DLB pathology than women, and women were more likely to have mixed pathology (DLB + $\mathrm{AD})[10,11]$. This is clinically relevant, as mixed pathology is linked to a more severe disease manifestation, with more cognitive disturbances, more frequent hallucinations, and shorter survival $[12,13]$. Although post-mortem studies are important, a major drawback is that they reflect end-stage disease. In contrast, cerebrospinal fluid (CSF) biomarkers enable in vivo studies in ongoing pathological processes. Previous CSF biomarker studies in AD have shown sex differences in CSF (p) Tau concentrations, suggesting a higher antemortem neurofibrillary tangle (NFT) load in women with AD [14-16]. Literature regarding sex differences in CSF biomarkers in DLB, however, is still limited. One small study reported lower levels of CSF alpha-synuclein in DLB women compared to DLB men, $\mathrm{AD}$, and controls [17]. In addition, apart from one study showing that visual hallucinations were more common in women [18], the relationship between clinical symptoms and sex in DLB has neither been studied extensively either. In order to provide biomarker-guided personalized medicine in the future, it is essential to further clarify sex differences in DLB biomarkers and symptomatology. Therefore, in the current study, we aimed to examine sex differences in CSF biomarkers and clinical symptomatology in a large welldefined cohort of DLB patients.

\section{Methods}

\section{Participants}

We included 223 DLB patients who visited the Alzheimer Center Amsterdam between 1999 and 2019 from the Amsterdam Dementia Cohort based on the availability of CSF [19]. All patients received standardized screening at baseline, which included a semi-structured medical history interview; physical, neurological, and neuropsychological examinations; magnetic resonance imaging (MRI); electroencephalography (EEG) or magnetoencephalography (MEG); and laboratory tests. Diagnoses were made in a multidisciplinary consensus meeting based on the results obtained from the standardized screening [19]. DLB patients were diagnosed clinically according to the current consensus criteria for probable DLB $[1,20]$. FP-CIT single-photon emission computed tomography (DATSPECT) was available in 103 patients and was positive for DLB diagnosis in 92 patients. DLB was confirmed at autopsy in 3 patients, of which 2 did not have a DAT-SPECT scan. Written informed consent was obtained from all study participants for the use of clinical data and biomaterial for research purposes.

\section{Measures \\ Clinical and cognitive features}

We rated the core clinical features at baseline from preformatted questionnaires or retrospectively from patients' medical charts. Hallucinations were scored according to the caregiver-rated neuropsychiatric inventory (NPI) that was available in 222 patients [21]. Parkinsonism was assessed with a preformatted checklist for extrapyramidal signs during the neurological exam (i.e., tremor, bradykinesia, and/or rigidity), data available for 216 patients. The semi-structured patient history interview was reviewed for information on fluctuations and RBD. Fluctuations were scored as being present when the patient or caregiver reported clear changes in attention and cognition during the day or between days. Information on fluctuations was available for 173 patients. REM sleep behavior disorder (RBD) was rated positive when caregivers reported that patients "act out" their dreams during their sleep. Information on RBD was available of 156 patients. Duration of complaints was systematically assessed during the patient history interview and was defined as the moment when the patient first noticed their cognitive complaints. Depressive symptoms were assessed using the 15-item Geriatric Depression Scale, with higher scores indicating more 
depressive symptoms [22]. Mini Mental State Examination (MMSE) was used to assess global cognition. Memory was assessed using the immediate and delayed recall of the Dutch version of the verbal learning test (RAVLT) [23]. Attention and speed was assessed using Trail Making Test (TMT)-A. Executive functioning was assessed using the ratio of TMT-B/TMT-A [24]. We calculated inverse scores for time-dependent tests (i.e., TMT-A and TMT-B), such that lower scores represent worse performance. Missing values of the TMT-B were imputed using the group ratio of TMT-B/TMT-A. Neuropsychological test scores were available for 205 patients, and test results were $Z$-transformed based on cognitively normal subjects $(n=533,60 \pm 10$ years, $54 \% \mathrm{~F}$, MMSE $=29 \pm 1$ ).

\section{Apolipoprotein E genotyping}

The QIAamp DNA blood isolation kit from Qiagen (Venlo, The Netherlands) was used to isolate DNA from $10-\mathrm{ml}$ vacutainer tubes containing EDTA. This was followed by genotype determination using the LightCycler ApoE Mutation Detection Kit (Roche Diagnostics, $\mathrm{GmbH}$, Mannheim, Germany). Subjects with at least one APOEe4 allele were defined as APOEe4 carriers, whereas no e4 allele defined subjects as APOEe4 non-carriers.

\section{CSF analysis}

For CSF collection and processing, standardized protocols were followed $[25,26]$. CSF total $\alpha$-syn concentrations were determined using a sandwich ELISA assay (developed by ADx NeuroSciences, Ghent, Belgium and commercialized by Euroimmun AG, Lübeck, Germany) [27]. To avoid blood contamination, $\alpha$-syn measures with high hemoglobuline $(\mathrm{Hb})$ concentrations (>10 ng/ $\mathrm{ml})$ or high erythrocyte count $(>500 \mathrm{ery} / \mathrm{ml})$ were excluded. CSF total $\alpha$-syn was available for 127 patients. CSF A $31-42$, Tau, and pTau concentrations were measured using a sandwich ELISA (Innotest, Fujirebio, Gent, Belgium), or the Elecsys A 342 , Tau, and pTau (181P) CSF assays run on the cobas e601 analyzer (Roche Diagnostics $\mathrm{GmbH}$ ). Elecsys values of A $\beta 42$, Tau, and pTau were converted to Innotest values using previously described formulas [28]. Drift corrected continuous A $\beta 42$ concentrations were used in the data analyses, and to define amyloid positivity, A $\beta 42$ concentrations were dichotomized using a cutoff $<813 \mathrm{pg} / \mathrm{ml}$ [29]. CSF A $\beta 1-$ 42 , Tau, and pTau were available for all patients.

\section{Statistical analysis}

Demographic data were compared between women and men using chi-squared tests, independent $t$ test, or Mann-Whitney $U$ tests where appropriate. All variables were checked for outliers and normality of residuals using Q-Q plots. TMT-data and CSF (p) Tau levels were log-transformed to meet assumptions of normality. Other outcome variables met the assumption of normally distributed residuals. To evaluate sex differences in clinical outcome measures, we used general linear models (GLM) with sex as the factor and baseline cognitive data or core clinical features as main outcome measures. Analyses were adjusted for age (clinical data) or age and education (cognitive data). Next, we performed general linear models (GLM) with sex as the factor and CSF biomarkers (i.e., $\alpha$-syn A $\beta 1-42$, Tau, and pTau) as the main outcome measure for each of the four CSF biomarkers separately. Analyses were adjusted for age (model 1) or age and APOEe4 genotype (model 2). A $p<0.05$ was considered significant. Analyses were conducted using $\mathrm{R}$ statistical program (version 3.5 .2 "eggshell igloo").

\section{Results}

Baseline characteristics are shown in Table 1. The majority of our sample consisted of men (f $17 \%$ vs $\mathrm{m} 83 \%$ ). Women were older than men (f 70 vs $\mathrm{m} 68 ; p=0.04$ ), exhibited a shorter duration of complaints before diagnosis (f 2.3 vs m 3.5, $p<0.001$ ), and more often reported visual hallucinations (f $58 \%$ vs $\mathrm{m} 38 \%, p<0.02$ ). Additionally, women more frequently carried the APOEe4 allele (f $70 \%$ vs $\mathrm{m} 52 \%, p=0.08$ ). No sex differences were found in other core clinical features, depressive symptoms, or years of education. After correcting for age and education, MMSE scores and animal fluency scores were lower in women than men. Performance on RAVLT (immediate and delayed recall), VAT, TMT-A, and TMT-B/ A did not differ between sexes (Table 1).

Next, we evaluated sex differences in CSF biomarker concentrations (Table 2; Fig. 1). Adjusted for age, women exhibited lower $\alpha$-syn concentrations compared to men. Moreover, women had lower A 342 concentrations than men and exhibited more often abnormal CSF amyloid concentrations (f $77 \%$ vs $\mathrm{m} 56 \%$, Table 1 ). No sex differences were found for CSF Tau or pTau concentrations. After adjusting for age and APOEe4 genotype, results for all CSF biomarkers remained largely similar (Table 2). When repeating the analyses in the subgroup with positive DAT-SPECT scans $(n=92$; f 15 (16\%), m $79(84 \%))$, we found similar effect sizes for all CSF biomarkers, although significance for $\alpha$-syn was lost due to the loss of power (eTable 1).

\section{Discussion}

In the present study, where DLB was more prevalent in men than in women, women had lower CSF A 342 and $\alpha$-syn concentrations. In addition, women had more frequent hallucinations, had shorter duration of complaints before diagnosis, were older, and had lower scores on MMSE and fluency-tasks. Based on our findings, one 
Table 1 Baseline characteristics

\begin{tabular}{|c|c|c|c|}
\hline & Women, $n=39$ & Men, $n=184$ & $\boldsymbol{p}$ value \\
\hline Age & $70.1 \pm 6.0$ & $67.7 \pm 7.3$ & 0.04 \\
\hline Education, years & $11.0 \pm 2.8$ & $11.4 \pm 2.9$ & 0.42 \\
\hline Duration of complaints, years & $2.3 \pm 1.2$ & $3.5 \pm 2.8$ & $<0.001$ \\
\hline APOE $\varepsilon 4$ carrier, $n$ (\%) & $22(70 \%)$ & $86(52 \%)$ & 0.08 \\
\hline Geriatric Depression Scale (0-15) & $4 \pm 3$ & $4 \pm 3$ & 0.65 \\
\hline \multicolumn{4}{|l|}{ Core clinical features, $\boldsymbol{n}(\%)$} \\
\hline Visual hallucinations, $n=222$ & $23(59 \%)$ & $69(38 \%)$ & 0.02 \\
\hline Parkinsonism, $n=216$ & $23(62 \%)$ & $130(73 \%)$ & 0.28 \\
\hline $\mathrm{RBD}, n=156$ & $11(55 \%)$ & $99(73 \%)$ & 0.17 \\
\hline Cognitive fluctuations, $n=173$ & $29(94 \%)$ & 119 (84\%) & 0.26 \\
\hline \multicolumn{4}{|l|}{ Cognitive test scores $^{\mathrm{a}}, n=205$} \\
\hline MMSE & $21 \pm 6$ & $23 \pm 4$ & 0.02 \\
\hline RAVLT_immediate recall & $-1.94 \pm 1.10$ & $-2.00 \pm 0.95$ & 0.74 \\
\hline RAVLT—delayed recall & $-1.80 \pm 0.98$ & $-1.72 \pm 0.96$ & 0.84 \\
\hline VAT-12 items & $-6.20 \pm 5.78$ & $-4.87 \pm 4.99$ & 0.31 \\
\hline TMT-A $^{\mathrm{b}}$ & $-5.47 \pm 6.46$ & $-4.60 \pm 4.84$ & 0.52 \\
\hline TMT-B/A ${ }^{b, c}$ & $-6.93 \pm 7.03$ & $-5.33 \pm 6.42$ & 0.32 \\
\hline Animal fluency & $-2.38 \pm 1.02$ & $-1.78 \pm 0.99$ & 0.002 \\
\hline \multicolumn{4}{|c|}{ Biomarker status, abnormal/available, $\boldsymbol{n}(\%)$} \\
\hline DAT SPECT, $n=107$ & 15/15 (100\%) & 79/91 (87\%) & 0.06 \\
\hline CSF A 442 status & $30 / 39(77 \%)$ & $104 / 186(56 \%)$ & 0.02 \\
\hline Abnormal Tau/Aß42 ratio (> 0.52) & $21 / 38(55 \%)$ & $55 / 185(30 \%)$ & 0.002 \\
\hline
\end{tabular}

Data represent mean $\pm \mathrm{SD}$, median [IQR] or $n(\%)$. Parkinsonism: bradykinesia, rigidity, or tremor

MMSE Mini Mental State Examination, RAVLT Raven auditory verbal learning test (Dutch version), VAT Visual Association Test, TMT Trail Making Test

aAdjusted for age and education. Data of RAVLT, VAT, TMT, and animal fluency represent $z$ scores, based on cognitively healthy subjects

${ }^{\mathrm{b}}$ Data are inverted and log-transformed

${ }^{\mathrm{C}}$ Missing values are imputed based on TMT-B/A ratio for $n=101$

could hypothesize that women have a more aggressive disease course compared to men.

When using predefined cutoffs for CSF A $\beta 42,77 \%$ of women had amyloid pathology, in contrast to $56 \%$ of men. This suggests that women more often had concomitant AD pathology, whereas men more often had pure DLB pathology. These in vivo findings are in line with previous autopsy studies that observed that men were more likely to die with pure DLB pathology and women more often died with DLB + AD pathology $[11,30]$. However, AD pathology is not only characterized by the presence of amyloid plaques, but also by NFT aggregation, which results in higher (p) Tau levels in CSF. As women in our study showed isolated lowered CSF A $\beta 42$ levels, an

Table 2 Cerebrospinal fluid concentrations at baseline

\begin{tabular}{|c|c|c|c|c|c|c|}
\hline & \multirow{2}{*}{$\begin{array}{l}\text { Women, } \\
n=39\end{array}$} & \multirow{2}{*}{$\begin{array}{l}\text { Men, } n= \\
184\end{array}$} & \multicolumn{2}{|l|}{ Model $1^{a}$} & \multicolumn{2}{|l|}{ Model $2^{\mathbf{b}}$} \\
\hline & & & Adjusted mean difference $\beta$ coefficient (95\%Cl) & $\boldsymbol{p}$ value & Adjusted mean difference $\beta$ coefficient (95\%Cl) & $\boldsymbol{p}$ value \\
\hline a-sync & $1558 \pm 431$ & $1895 \pm 707$ & $-390(-731:-48)$ & 0.03 & $-309(-657:-40)$ & 0.08 \\
\hline$A \beta 42$ & $696 \pm 210$ & $824 \pm 251$ & $-108(-190:-26)$ & 0.01 & $-103(-191:-14)$ & 0.02 \\
\hline $\mathrm{Tau}^{\mathrm{d}}$ & $5.9 \pm 0.6$ & $5.7 \pm 0.5$ & $0.10(-0.09:-0.29)$ & 0.31 & $0.06(-0.14:-0.03)$ & 0.55 \\
\hline pTau $^{\text {d }}$ & $3.9 \pm 0.5$ & $3.9 \pm 0.5$ & $0.03(-0.13: 0.19)$ & 0.74 & $0.01(-0.15: 0.17)$ & 0.90 \\
\hline
\end{tabular}

Data presented as mean \pm SD

${ }^{a}$ Model 1: adjusted for age

${ }^{b}$ Model 2: adjusted for age and APOEe4 genotype

ca-syn results were available for $n=117(f=17 ; m=100)$

${ }^{\mathrm{d}}$ Analyses were performed with LN transformed data to meet assumptions of normality 


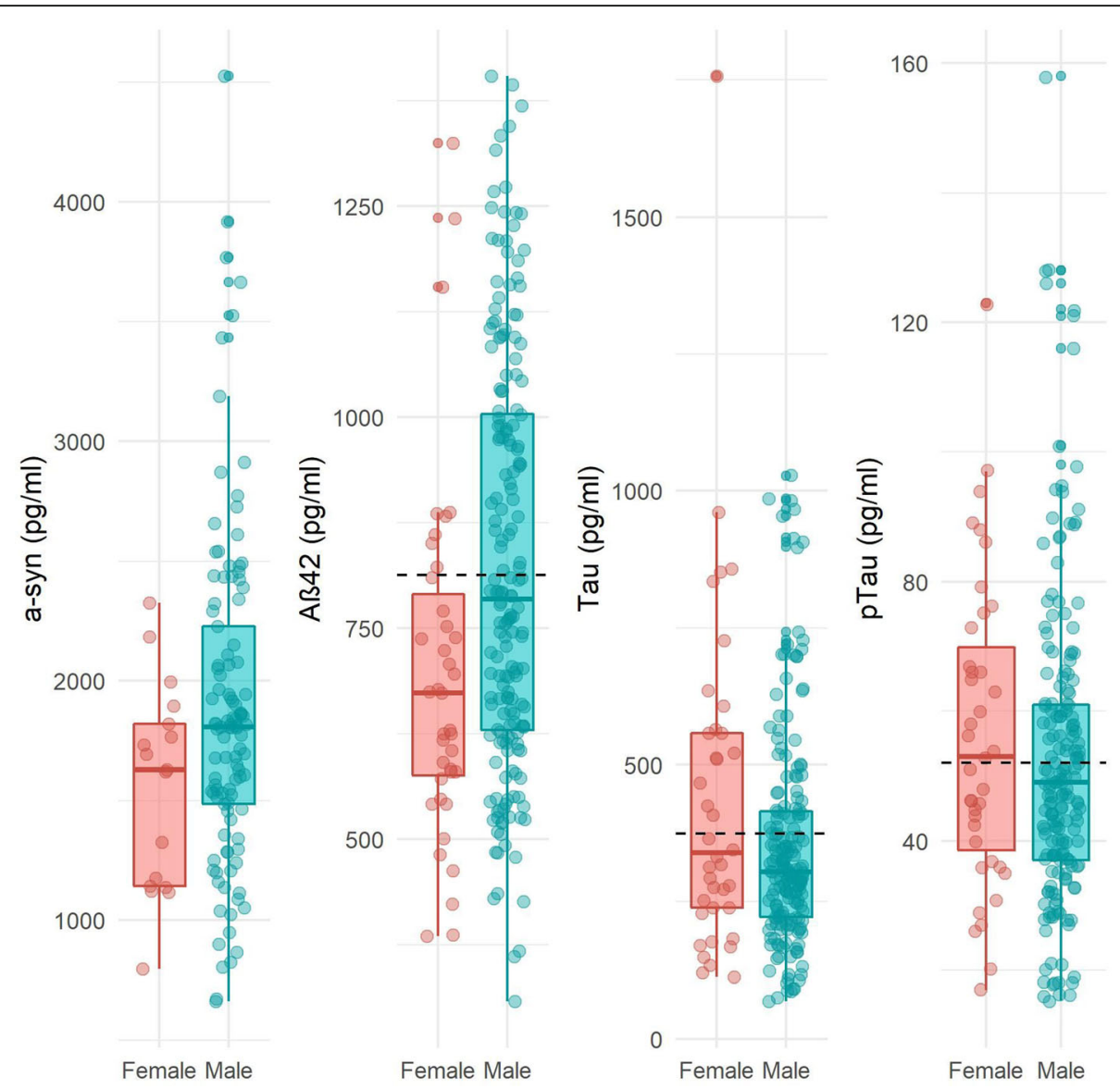

Fig. 1 Sex differences in cerebrospinal fluid biomarker concentrations. Mean differences in CSF biomarker concentrations in picorgrams/milliliter between women and men. ${ }^{*} p<0.05$. Abbreviations: CSF, cerebrospinal fluid; $a-s y n$; alpha-synuclein; A $\beta 42 ;$ amyloid- $\beta 42 ;$ Tau, total tau; ( $p$ ) Tau, tau phosphorylated at threonine 181. Dashed line reflects cutoffs for Aß42 (813 pg/ml), Tau (375 pg/ml), and (p) Tau (52 pg/ml)

alternative explanation could be that the lower levels of A 342 are directly related to the DLB disease process, rather than reflecting concomitant AD pathology. Previous studies have shown that $A \beta 42$ induces the formation of high-molecular-weight $\alpha$-syn, and vice versa [31]. Therefore, this synergistic interaction between $\mathrm{A} \beta 42$ and $\alpha$-syn could lead to increased aggregation and accumulation of both $\alpha$-syn and amyloid pathology in the women in our study compared to men [31-33].

Concomitant amyloid pathology in DLB has previously been related to a more severe clinical disease burden [12]. In line with these former findings, women in our cohort had lower MMSE and fluency test scores, and more frequent hallucinations, but a shorter duration of symptoms before diagnosis, suggesting a more aggressive disease course upon diagnosis in women with DLB. This could well be related to the amyloid-pathology that is more often encountered in DLB women. In our study, duration of complaints was estimated retrospectively; therefore, we cannot completely rule out that women with DLB were already in a later disease stage at the moment of diagnosis. Consistent with our hypothesis of a more aggressive disease course in women, one other study found that women with DLB have shorter survival after onset of dementia [34]. Prospective studies with longitudinal data are needed to further investigate sex differences in disease course over time.

Our data are in line with former observations of a strong male-predominance in DLB, as $83 \%$ of patients in our cohort were men [4-7]. The strong malepredominance in DLB could be explained by hormonal differences between sexes. Several previous studies have shown that $17 \beta$-estradiol (E2) conveys neuroprotective effects in women [35]. In fact, a previous in vitro study has shown that estradiol exhibits dose-dependent inhibition of $\alpha$-syn aggregation and destabilization of preformed $\alpha$-syn, which is thought to be driven by the antioxidant properties of estrogens [36-39]. This suggests that until menopause occurs in women, at the average age of 55 years, they are protected against the development of diseases such as DLB and Parkinson's disease. During the second half of menopause, however, 
women show a sudden drop of estradiol levels [40], thus triggering the aggregation of $\alpha$-syn and impeding the breakdown of preformed $\alpha$-syn. If this is the case, sex differences in prevalence are expected to be more balanced with increasing age. In line with this hypothesis, a recent study reported a higher DLB prevalence of men than women under the age of 75 years, which equalized between the ages of 75 and 80 years. Above the age of 80 years, however, DLB was more common in women [8]. Future studies will have to demonstrate whether this shift in prevalence is mediated by hormonal differences.

Another explanation for the higher prevalence of DLB in men could be that concomitant $\mathrm{AD}$ pathology seen in DLB women potentially introduces a bias towards diagnosing these women with AD rather than DLB, therefore underdiagnosing the true proportion of women with DLB. In line with this reasoning, one study found that concomitant AD pathology decreased diagnostic accuracy, as typical AD symptoms were more pronounced, in contrast to DLB symptoms [41]. Our results imply that clinicians should be conscious of the risk of underdiagnosing women with DLB.

Our study has several strengths, including our welldefined, large sample of DLB patients. The clinical diagnosis of DLB was largely supported by DAT-SPECT imaging. In addition, we had CSF $\alpha$-syn available in a considerable part of patients and CSF AD biomarkers in all patients. Furthermore, CSF collection follows a highly standardized protocol, assuring that the possibility of pre-analytical confounders is minimized [19]. Among the limitations is the retrospective design of our study. Information on RBD and fluctuations was rated retrospectively based on patients' medical charts. In some cases, these features were not reported, which could either mean that they were not present or that it was not asked by the medical doctor. Therefore, there was a relatively large number of missing values for these features and there is a risk of underreporting. Future prospective studies should use standardized methods to assess these symptoms. A second limitation is that CSF total $\alpha$-syn is not yet validated as a clinically useful marker in DLB, and there might be differences in sensitivity between different $\alpha$-syn species. For example, soluble $\alpha$-syn oligomers could be more useful in DLB, because oligomeric forms of $\alpha$-syn seem to play a more essential role in the pathogenesis of $\alpha$-synucleinopathies than $t-\alpha$-syn [42]. Another $\alpha$-syn species of interest is $\alpha$ syn phosphorylated at serine 129 (pSer129- $\alpha$-syn), as approximately $90 \%$ of accumulated $\alpha$-syn in Lewy bodies consists of pSer129- $\alpha$-syn [43]. However, these species cannot yet be measured robustly with commercial assays as these have not yet been validated. A last limitation was that a small part of our patients had a normal DATSPECT scan, not supportive of DLB diagnosis. However, clinical diagnosis was made carefully in a tertiary memory clinic setting and false-negative DAT-SPECT scans in early disease stages have been previously described [44].

\section{Conclusions}

To conclude, our results indicate sex differences in DLB, wherein concomitant $\mathrm{AD}$ pathology was more frequent in women and CSF alpha-synuclein levels were lower in women compared to men. Concomitant pathology, combined with lower cognitive test scores and a shorter duration of complaints before diagnosis, could suggest a higher disease severity in women. Our results raise awareness among clinicians of the possibility that women are underdiagnosed and have the potential to aid in defining sex-specific personalized medicine strategies within DLB.

\section{Supplementary information}

Supplementary information accompanies this paper at https://doi.org/10 1186/s13195-020-00610-9.

Additional file 1: eTable 1. Cerebrospinal fluid concentrations at baseline in DAT SPECT positive subgroup.

\section{Abbreviations \\ DLB: Dementia with Lewy bodies; CSF: Cerebrospinal fluid; Aß: Amyloid- $\beta$; Aß42: Amyloid $\beta 1-42 ;$ a-syn: Alpha-synuclein; Tau: Total tau; pTau: Tau phosphorylated at threonine 181; CV: Coefficient of variation; MMSE: Mini Mental State Examination; GLM: General linear models; RBD: REM sleep behavior disorder; NFT: Neurofibrillary tangles; AD: Alzheimer's disease; MRI: Magnetic resonance imaging; EEG: Electroencephalography; DAT- SPECT: FP-CIT single-photon emission computed tomography; NPI: Neuropsychiatric inventory; TMT: Trail Making Test; RAVLT: Verbal learning test; Hb: Hemoglobuline}

\section{Acknowledgements}

Thanks to Cindy Francois (ADx, Neurosciences) for the preparation of the $\mathrm{Hb}$ ELISA kits.

\section{Authors' contributions}

$\mathrm{MvdB}$ and RBM were equally responsible for the study design, analysis and interpretation of data, and the writing of the manuscript. IvS was responsible for the study concept and design, review of the statistical analysis, and critical revision of the manuscript for important intellectual content. HvdS was responsible for critical revision of the manuscript for important intellectual content. PS was responsible for critical revision of the manuscript for important intellectual content. CET was responsible for critical revision of the manuscript for important intellectual content. AWL was responsible for the study concept and design and critical revision of the manuscript for important intellectual content. WMvdF was responsible for the study concept and design, the review of the statistical analysis, and the critical revision of the manuscript for important intellectual content. The authors read and approved the final manuscript.

\section{Funding}

Research of the Alzheimer Center Amsterdam is part of the

Neurodegeneration research program of the Neuroscience Campus Amsterdam. The Alzheimer Center Amsterdam is supported by Alzheimer Nederland and Stichting VUMC funds. A.L. has received funding from stichting Dioraphte and ZonMW Memorabel (project \#733050509).

\section{Availability of data and materials}

All data used and/or analyzed during the present study are available from the corresponding author on reasonable request. 


\section{Ethics approval and consent to participate}

Written informed consent was obtained from all study participants for the use of clinical data and biomaterial for research purposes. This study was approved by the local medical ethical committee of the Amsterdam UMC, location VUmc.

\section{Consent for publication}

Not applicable.

\section{Competing interests}

Prof. Dr. Teunissen has a collaboration contract with ADx Neurosciences and performed contract research or received grants from Probiodrug, AC Immune, Biogen-Esai, CogRx, Toyama, Janssen prevention center, Boehringer, AxonNeurosciences, Fujirebio, EIP farma, PeopleBio, and Roche. Prof. Dr. Scheltens has acquired grant support (for the institution) from GE Healthcare and Piramal. In the past 2 years, he has received consultancy/speaker fees (paid to the institution) from Novartis, Probiodrug, Biogen, Roche, and EIP Pharma, LLC. Dr. Lemstra has received funding from stichting Dioraphte and ZonMW Memorabel (project \#733050509). Research programs of Prof. Dr. Wiesje van der Flier have been funded by ZonMW, NWO, EU-JPND, Alzheimer Nederland, CardioVascular Onderzoek Nederland, Health Holland, Topsector Life Sciences \& Health, stichting Dioraphte, Gieskes-Strijbis fonds, stichting Equilibrio, Biogen MA Inc., Life-MI, AVID, and Combinostics. WF holds the Pasman chair. WF has performed contract research for Biogen MA Inc. All funding is paid to her institution. Van de Beek, Babapour Mofrad, van Steenoven, Dr. vanderStichele report no disclosures relevant to this manuscript.

\section{Author details \\ ${ }^{1}$ Alzheimer Center Amsterdam \& Department of Neurology, Neuroscience Campus Amsterdam Neuroscience, Vrije Universiteit Amsterdam, Amsterdam UMC, VU University Medical Center, Amsterdam, The Netherlands. ${ }^{2}$ Neurochemistry Laboratory and Biobank, Department of Clinical Chemistry, Amsterdam Neuroscience, Amsterdam UMC, VU University Medical Center, Amsterdam, The Netherlands. ${ }^{3}$ Biomarkable bvba, Ghent, Belgium. ${ }^{4}$ Department of Epidemiology and Biostatistics, Vrije Universiteit Amsterdam, Amsterdam UMC, VU University Medical Center, Amsterdam, The Netherlands.}

\section{Received: 15 October 2019 Accepted: 31 March 2020} Published online: 17 April 2020

\section{References}

1. McKeith IG, Boeve BF, Dickson DW, Halliday G, Taylor J-P, Weintraub D, et al. Diagnosis and management of dementia with Lewy bodies fourth consensus report of the DLB consortium. Neurology. 2017;89(1):88-100.

2. Vekrellis $K$, Xilouri $M$, Emmanouilidou E, Rideout HJ, Stefanis L. Pathological roles of a-synuclein in neurological disorders. Lancet Neurol. 2011;10(11):1015-25.

3. van Steenoven I, Aarsland D, Weintraub D, Londos E, Blanc F, van der Flier WM, et al. Cerebrospinal fluid Alzheimer's disease biomarkers across the Spectrum of Lewy body diseases: results from a large multicenter cohort. J Alzheimers Dis. 2016;54(1):287-95.

4. Kane JP, Surendranathan A, Bentley A, Barker SA, Taylor J-P, Thomas AJ, et al. Clinical prevalence of Lewy body dementia. Alzheimers Res Ther. 2018;10(1):19.

5. Fereshtehnejad S-M, Religa D, Westman E, Aarsland D, Lökk J, Eriksdotter M. Demography, diagnostics, and medication in dementia with Lewy bodies and Parkinson's disease with dementia: data from the Swedish dementia quality registry (SveDem). Neuropsychiatr Dis Treat. 2013;9:927.

6. Savica R, Grossardt BR, Bower JH, Boeve BF, Ahlskog JE, Rocca WA. Incidence of dementia with Lewy bodies and Parkinson disease dementia. JAMA Neurol. 2013;70(11):1396-402.

7. Podcasy $\mathrm{J}$, Epperson CN. Considering sex and gender in Alzheimer disease and other dementias. Dialogues Clin Neurosci. 2016;18(4):437.

8. Mouton A, Blanc F, Gros A, et al. Sex ratio in dementia with Lewy bodies balanced between Alzheimer's disease and Parkinson's disease dementia: a cross-sectional study. Alz Res Therapy. 2018;10:92. https://doi.org/10.1186/ s13195-018-0417-4.

9. Price A, Farooq R, Yuan J-M, Menon VB, Cardinal RN, O'Brien JT. Mortality in dementia with Lewy bodies compared with Alzheimer's dementia: a retrospective naturalistic cohort study. BMJ Open. 2017;7(11).
10. Nelson PT, Jicha GA, Kryscio RJ, Abner EL, Schmitt FA, Cooper G, et al. Low sensitivity in clinical diagnoses of dementia with Lewy bodies. J Neurol. 2010;257(3):359-66.

11. Barnes LL, Lamar M, Schneider JA. Sex differences in mixed neuropathologies in community-dwelling older adults. Brain Res. 2019;1719:11-6.

12. Lemstra AW, de Beer MH, Teunissen $C E$, Schreuder $C$, Scheltens $P$, van der Flier WM, et al. Concomitant AD pathology affects clinical manifestation and survival in dementia with Lewy bodies. J Neurol Neurosurg Psychiatry. 2017;88(2):113-8.

13. Abdelnour C, van Steenoven I, Londos E, Blanc F, Auestad B, Kramberger $M G$, et al. Alzheimer's disease cerebrospinal fluid biomarkers predict cognitive decline in Lewy body dementia. Mov Disord. 2016;31(8):1203-8.

14. Hohman TJ, Dumitrescu L, Barnes LL, Thambisetty M, Beecham G, Kunkle B, et al. Sex-specific association of apolipoprotein e with cerebrospinal fluid levels of tau. JAMA Neurol. 2018;75(8):989-98.

15. Barnes LL, Wilson RS, Bienias JL, Schneider JA, Evans DA, Bennett DA. Sex differences in the clinical manifestations of Alzheimer disease pathology. Arch Gen Psychiatry. 2005;62(6):685-91.

16. Mofrad RB, van der Flier WM. Nature and implications of sex differences in AD pathology. Nat Rev Neurol. 2019;15(1):6.

17. Wennström M, Londos E, Minthon L, Nielsen HM. Altered CSF orexin and asynuclein levels in dementia patients. J Alzheimers Dis. 2012;29(1):125-32.

18. Chiu PY, Teng PR, Wei CY, Wang CW, Tsai CT. Gender difference in the association and presentation of visual hallucinations in dementia with Lewy bodies: a cross-sectional study. Int J Geriatr Psychiatry. 2018;33(1):193-9.

19. van der Flier WM, Scheltens P. Amsterdam dementia cohort: performing research to optimize care. J Alzheimers Dis. 2018;62(3):1091-111.

20. McKeith I, Dickson DW, Lowe J, Emre M, O'brien J, Feldman H, et al. Diagnosis and management of dementia with Lewy bodies third report of the DLB consortium. Neurology. 2005;65(12):1863-72.

21. Cummings $\mathrm{J}$. The neuropsychiatric inventory assessing psychopathology in dementia patients. Neurology. 1997:48(5 Suppl 6):10S-6S.

22. Sheikh Jl, Yesavage JA. Geriatric depression scale (GDS): recent evidence and development of a shorter version. Clinical Gerontologist. J Aging Mental Health. 1986:5(1-2):165-73.

23. Saan R, Deelman B. De 15-woordentest A en B (een voorlopige handleiding). Groningen: Afdeling Neuropsychologie, AZG; 1986.

24. Reitan RM. Validity of the Trail Making Test as an indicator of organic brain damage. Percept Mot Skills. 1958;8(3):271-6.

25. Mofrad RB, Bouwman FH, Slot RE, Timmers T, van der Flier WM, Scheltens $P$, et al. Lumbar puncture in patients with neurologic conditions. Alzheimers Dement. 2017:8:108-10.

26. Del Campo M, Mollenhauer B, Bertolotto A, Engelborghs S, Hampel H, Simonsen $\mathrm{AH}$, et al. Recommendations to standardize preanalytical confounding factors in Alzheimer's and Parkinson's disease cerebrospinal fluid biomarkers: an update. Biomark Med. 2012;6(4):419-30.

27. Chiasserini D, Biscetti L, Eusebi P, Salvadori N, Frattini G, Simoni S, et al. Differential role of CSF fatty acid binding protein 3, a-synuclein, and Alzheimer's disease core biomarkers in Lewy body disorders and Alzheimer's dementia. Alzheimers Res Ther. 2017;9(1):52.

28. Willemse EA, van Maurik IS, Tijms BM, Bouwman FH, Franke A, Hubeek I, et al. Diagnostic performance of Elecsys immunoassays for cerebrospinal fluid Alzheimer's disease biomarkers in a nonacademic, multicenter memory clinic cohort: the ABIDE project. Alzheimers Dement. 2018;10:563-72.

29. Tijms BM, Willemse EA, Zwan MD, Mulder SD, Visser PJ, van Berckel BN, et al. Unbiased approach to counteract upward drift in cerebrospinal fluid amyloid- $\beta$ 1-42 analysis results. Clin Chem. 2017;2017:281055.

30. Nelson PT, Schmitt FA, Jicha GA, Kryscio RJ, Abner EL, Smith CD, et al. Association between male gender and cortical Lewy body pathology in large autopsy series. J Neurol. 2010;257(11):1875-81.

31. Clinton LK, Blurton-Jones M, Myczek K, Trojanowski JQ, LaFerla FM. Synergistic interactions between $A \beta$, tau, and $\alpha$-synuclein: acceleration of neuropathology and cognitive decline. J Neurosci. 2010;30(21):7281-9.

32. Irwin DJ, Lee VM-Y, Trojanowski JQ. Parkinson's disease dementia: convergence of a-synuclein, tau and amyloid- $\beta$ pathologies. Nat Rev Neurosci. 2013;14(9):626.

33. Colom-Cadena M, Gelpi E, Charif S, Belbin O, Blesa R, Martí MJ, et al. Confluence of a-synuclein, tau, and $\beta$-amyloid pathologies in dementia with Lewy bodies. J Neuropathol Exp Neurol. 2013;72(12):1203-12.

34. Williams MM, Xiong C, Morris JC, Galvin JE. Survival and mortality differences between dementia with Lewy bodies vs Alzheimer disease. Neurology. 2006;67(11):1935-41. 
35. Liu B, Dluzen DE. Oestrogen and nigrostriatal dopaminergic neurodegeneration: animal models and clinical reports of Parkinson's disease. Clin Exp Pharmacol Physiol. 2007;34(7):555-65.

36. Hirohata M, Ono K, Morinaga A, Ikeda T, Yamada M. Anti-aggregation and fibril-destabilizing effects of sex hormones on a-synuclein fibrils in vitro. Exp Neurol. 2009;217(2):434-9.

37. Zhu M, Rajamani S, Kaylor J, Han S, Zhou F, Fink AL. The flavonoid baicalein inhibits fibrillation of a-synuclein and disaggregates existing fibrils. J Biol Chem. 2004;279(26):26846-57.

38. Ono K, Yamada M. Antioxidant compounds have potent anti-fibrillogenic and fibril-destabilizing effects for a-synuclein fibrils in vitro. J Neurochem. 2006;97(1):105-15.

39. Ehrnhoefer DE, Bieschke J, Boeddrich A, Herbst M, Masino L, Lurz R, et al. EGCG redirects amyloidogenic polypeptides into unstructured, off-pathway oligomers. Nat Struct Mol Biol. 2008:15(6):558.

40. Mielke MM, Vemuri P, Rocca WA. Clinical epidemiology of Alzheimer's disease: assessing sex and gender differences. Clin Epidemiol. 2014;6:37.

41. Merdes A, Hansen L, Jeste D, Galasko D, Hofstetter C, Ho G, et al. Influence of Alzheimer pathology on clinical diagnostic accuracy in dementia with Lewy bodies. Neurology. 2003;60(10):1586-90.

42. van Steenoven I, Majbour NK, Vaikath NN, Berendse HW, van der Flier WM, van de Berg WD, et al. a-Synuclein species as potential cerebrospinal fluid biomarkers for dementia with lewy bodies. Mov Disord. 2018;33(11):1724-33.

43. Anderson JP, Walker DE, Goldstein JM, De Laat R, Banducci K, Caccavello RJ, et al. Phosphorylation of Ser-129 is the dominant pathological modification of a-synuclein in familial and sporadic Lewy body disease. J Biol Chem. 2006;281(40):29739-52

44. van der Zande JJ, Booij J, Scheltens P, Raijmakers PG, Lemstra AW. [(123)]FPCIT SPECT scans initially rated as normal became abnormal over time in patients with probable dementia with Lewy bodies. Eur I Nucl Med Mol Imaging. 2016;43(6):1060-6.

\section{Publisher's Note}

Springer Nature remains neutral with regard to jurisdictional claims in published maps and institutional affiliations.

Ready to submit your research? Choose BMC and benefit from:

- fast, convenient online submission

- thorough peer review by experienced researchers in your field

- rapid publication on acceptance

- support for research data, including large and complex data types

- gold Open Access which fosters wider collaboration and increased citations

- maximum visibility for your research: over $100 \mathrm{M}$ website views per year

At $\mathrm{BMC}$, research is always in progress.

Learn more biomedcentral.com/submissions 